\title{
DINÂMICA VEGETACIONAL EM PASTAGEM NATURAL SUBMETIDA A TRATAMENTOS DE QUEIMA E PASTEJO
}

\author{
VEGETATION DYNAMICS OF NATURAL GRASSLAND UNDER TREATMENTS OF BURNING \\ AND GRAZING
}

\author{
Fernando Luiz Ferreira de Quadros ${ }^{1}$ Valério De Patta Pillar $^{2}$
}

RESUMO

Foram avaliados durante três anos os efeitos de tratamentos de fogo e pastejo sobre a dinâmica da vegetação de uma pastagem natural localizada em Santa Maria, na região da Depressão Central, Rio Grande do Sul. Foi considerada a hipótese de resiliência, resultado das espécies componentes da pastagem terem evoluído sob influência de tais distúrbios. O experimento foi composto por oito parcelas experimentais submetidas a combinações de níveis de pastejo (pastejado, excluído) e de fogo (queimado, não-queimado), em duas posições de relevo (encosta, baixada). A análise multivariada dos dados de composição de espécies foi baseada em ordenação e testes de aleatorização. A vegetação sob efeito de pastejo, independente da queima, apresentou trajetórias direcionais, enquanto sob exclusão as trajetórias foram caóticas. $O$ efeito do pastejo parece ser determinante da dinâmica vegetacional $(P=0,077)$.

Palavras-chave: trajetórias de vegetação, composição florística, resiliência, ecologia.

\section{SUMMARY}

The effect of fire and grazing treatments on vegetation dynamics was evaluated during three years on a natural grassland located in Santa Maria, in the region of "Depressão Central", Rio Grande do Sul, Brazil. A hypothesis of resilience resulting from the fact that the species of the grassland evolved under the influence of these disturbances was considered. The experimental setup was formed by eight plots subjected to combinations of grazing (grazed, ungrazed) and fire (burned, unburned) levels, on two relief positions (convex, concave slope). Multivariate analysis of compositional data used ordination and randomization testing. Vegetation under grazing tended to show directional trajectories of floristic composition change, while under grazing exclusion the trajectories could be considered chaotic, independently from the plots being burned or not. Grazing effect seems to be determinant of vegetacional dynamics $(P=0.077)$.

\author{
Key words: vegetation trajectories, floristic composition, \\ resilience, ecology.
}

\section{INTRODUÇÃO}

O desenvolvimento de sistemas de produção viáveis economicamente, que tornem compatíveis bons ganhos animais com a preservação dos ecossistemas de pastagens naturais, é apoiado pelo conhecimento da dinâmica da vegetação que ocorre após determinadas práticas de manejo. Ademais, essa tarefa também é apoiada pelo entendimento da fisiologia e morfologia das espécies forrageiras e sua resposta a alterações naturais ou antropogênicas de fatores ambientais (NABINGER, 1980; FONTANELI \& JACQUES, 1988).

A importância do fogo na origem e na manutenção das áreas de pastagens no mundo é bastante discutida. Alguns historiadores chegam a afirmar que a causa primária para o desenvolvimento das pastagens e sua manutenção foi o fogo e não o clima. Segundo VALENTINE (1974), esta visão é extrema; o fogo é obviamente um importante fator ambiental e, provavelmente, nenhum ecossistema de pastagem tenha se desenvolvido sem a influência do fogo. No Brasil, tanto a comunidade científica quanto a legislação ambiental, ainda, são comandadas por conceitos conservacionistas florestais que estão longe de refletir a realidade das pastagens naturais, sobre as quais ainda há uma carência de dados quantitativos.

\footnotetext{
${ }^{1}$ Engenheiro Agrônomo, Doutor, Professor Adjunto, Departamento de Zootecnia, Universidade Federal de Santa Maria (UFSM), 97105900, Santa Maria, RS. E-mail: fquadros@ ccr.ufsm.br. Autor para correspondência.

${ }^{2}$ Engenheiro Agrônomo, PhD., Professor Titular, Departamento de Ecologia, Universidade Federal do Rio Grande do Sul. Bolsista do CNPq.
} 
Há divergências nas interpretações de resultados de pesquisa sobre o fogo em vegetação natural (CASTILHOS \& JACQUES, 1985; DÜRR et al., 1993; DAMÉ et al., 1997). Isto pode ser atribuído ao fato de serem utilizados pressupostos de análise estatística dos dados que foram desenvolvidos para vegetação homogênea, especialmente para monoculturas, com um comportamento muito diverso dos padrões de distribuição exibidos pelas espécies naturais. Com exceção do trabalho de EGGERS \& PORTO (1994), todas as demais análises consideraram $\mathrm{O}$ comportamento isolado das principais espécies, sem incluir a possibilidade de interação entre os componentes, que nos permite a análise multivariada.

Diante da escassez de informações quantitativas para as condições de pastagens naturais no Sul do Brasil, bem como de resultados conflitantes sobre os efeitos do fogo, aliados às dificuldades de interpretação e identificação das condicionantes destes efeitos, o presente trabalho procura estabelecer relações entre a queima e a estrutura da vegetação campestre.

Considerando-se, neste artigo, a hipótese de resiliência da vegetação campestre aos distúrbios de fogo e pastejo. A vegetação campestre do Cone Sul provavelmente evoluiu sob presença do fogo e do pastejo, como elementos de distúrbio. Conjeturase que a intensidade do fogo foi maior do que do pastejo na história evolutiva das plantas dos campos, o que poderia ter determinado o desenvolvimento de mecanismos de resiliência mais efetivos em resposta ao fogo. A ação antrópica provavelmente aumentou a freqüência de ambos os elementos e, mais recentemente (há cerca de 150 anos), o aumento da intensidade do pastejo determinou a redução da intensidade de queimadas, tempo insuficiente para o desenvolvimento de mecanismos adaptativos (PILLAR \& QUADROS, 1997).

Este trabalho apresenta resultados experimentais avaliando efeitos de queima e pastejo sobre a dinâmica da composição florística de uma pastagem natural em Santa Maria, RS.

\section{MATERIAL E MÉTODOS}

O experimento foi realizado em área experimental do Departamento de Zootecnia, no Campus da Universidade Federal de Santa Maria, entre as coordenadas de $29^{\circ} 45^{\prime} \mathrm{S}$ e $53^{\circ} 45^{\prime} \mathrm{W}$. A altitude média é de $95 \mathrm{~m}$ acima do nível do mar e o clima é subtropical úmido. $\mathrm{O}$ solo da área experimental é desenvolvido sobre o fácies Alemoa da formação Santa Maria, textura média, de relevo suave-ondulado, substrato siltito-arenito, sendo classificado como muito ácido $(\mathrm{pH}$ entre 5,0-5,5). Os solos das posições de relevo encosta e baixada foram classificados, respectivamente, como Podzólico bruno acinzentado distrófico com argila de atividade baixa e Planossolo eutrófico com argila de atividade alta.

A área experimental tem sido utilizada, historicamente, como invernada para cria e recria de bovinos de corte. Em função da baixa carga, a área apresentava um aspecto de campo macegoso, com predomínio de Erianthus angustifolius, segundo depoimentos dos responsáveis pelo manejo desde os anos 70. Com o crescimento populacional do rebanho, houve um crescente aumento da carga que alterou a fisionomia da pastagem, para uma dominância de Andropogon lateralis, conforme comprovam os levantamentos de KERSTING (1994) e DAMÉ et al.(1997), em área próxima ao experimento. Não existem registros e nem indícios, a campo, de que a área tenha sofrido algum tipo de preparo de solo, para fins agrícolas, podendo-se supor que seja uma pastagem natural há centenas de anos

Em um potreiro de aproximadamente 40ha, diferido desde março de 1995, foram demarcadas quatro transecções de $15 \mathrm{~m}$ por posição de relevo (encosta, baixada). As transecções foram localizadas de forma a apresentar vegetação aparentemente homogênea, entre transecções na mesma posição de relevo, o que foi facilitado pela forma estreita e longa da parcela. Em cada transecção demarcaram-se 30 quadros contíguos de $0,5 \mathrm{~m}$ de lado. $\mathrm{O}$ conjunto de 30 quadros em uma transecção constitui uma parcela experimental de 0,5 $\mathrm{x} 15 \mathrm{~m}$, a qual foi submetida a um dos tratamentos abaixo definidos. Os quadros foram identificados por pinos de ferro galvanizado, em forma de L, em cada canto da diagonal.

Os tratamentos foram definidos pela combinação de dois fatores, queima da vegetação e exclusão do pastejo, constituindo quatro tratamentos assim dispostos: 1) Queimado e excluído do pastejo; 2) Queimado e pastejado; 3) Não queimado e excluído do pastejo; 4) Não queimado e pastejado. As repetições constituíram dois blocos (encosta e baixada). As parcelas excluídas ficaram em uma área de dois hectares cercada em julho de 1995, enquanto as parcelas não excluídas permaneceram sob pastejo.

Cada quadro de $0,5 \times 0,5 \mathrm{~m}$ foi descrito em sua composição florística, através de listagem das espécies componentes e respectivas quantidades de biomassa obtidas por estimativa visual. A avaliação 
da disponibilidade total de matéria seca, da participação relativa dos principais componentes e da freqüência relativa dos demais componentes da vegetação nativa foi realizada, através de dupla amostragem, seguindo os procedimentos de campo do Software Botanal (TOTHILL et al., 1992). Os procedimentos foram modificados para incluir todas as espécies presentes, sendo que aquelas que apresentaram menos de $5 \%$ da matéria seca disponível tiveram sua participação estimada homogeneamente em uma unidade percentual para cada espécie.

As avaliações foram realizadas de agosto de 1995 a dezembro de 1997 . O primeiro inventário foi realizado em agosto (14 a 18/8), apenas nas transecções que seriam queimadas em $19 / 8 / 95$, sendo as demais avaliadas em novembro (9 a 17/11), devido à maior facilidade para identificação das espécies. A segunda avaliação foi realizada de 11 a 16/01/ 96, nas transecções queimadas, e de 02 a 05/02/96, nas unidades amostrais não queimadas. $\mathrm{O}$ terceiro inventário ocorreu no período de 9 a $18 / 12 / 96$ e o quarto foi executado de 8/12/97 a 19/12/97.

Os dados foram submetidos à análise multivariada utilizando os softwares Multiv (PILLAR, 1997), SYNCSA (PILLAR \& ORLÓCI, 1993). Os dados de biomassa por unidade amostral foram padronizados pelos totais marginais, quando a medida de semelhança utilizada nas comparações foi a distância euclidiana. Em outras análises, foi utilizada a distância de corda. Os dados foram submetidos à análise de ordenação por coordenadas principais para identificar as tendências principais de variação e observar as trajetórias de cada parcela experimental. Os tratamentos (fatores principais e interações) foram comparados através de análise de variância com testes de aleatorização (PILLAR \& ORLÓCI, 1996). Devido à baixa correlação espacial observada entre quadros de $0,25 \mathrm{~m}^{2}$ nas transecções, as parcelas de $0,5 \times 15 \mathrm{~m}$ foram divididas em três parcelas de $0,5 \mathrm{~m} \times 3 \mathrm{~m}$, para aumentar o número de combinações possíveis nas iterações do teste.

\section{RESULTADOS E DISCUSSÃO}

A análise foi realizada com a composição florística das parcelas experimentais (média dos 30 quadros amostrais em cada transecção) nas diferentes épocas de avaliação. Foram assim definidos trinta e dois inventários localizados no tempo e no espaço. Para facilitar a visualização das trajetórias em diagramas de ordenação, as parcelas são apresentadas em diagramas separados, segundo os regimes de pastejo e o efeito do fogo (Figura 1).

Pode-se observar que as parcelas queimadas, na encosta, apresentam trajetórias paralelas, que iniciam com uma dominância de Andropogon lateralis e ao longo do tempo aumenta a participação de Paspalum notatum e Vernonia nudiflora, particularmente no tratamento pastejado, que apresentou a trajetória mais longa. A trajetória do tratamento queimado e excluído, na encosta (a),

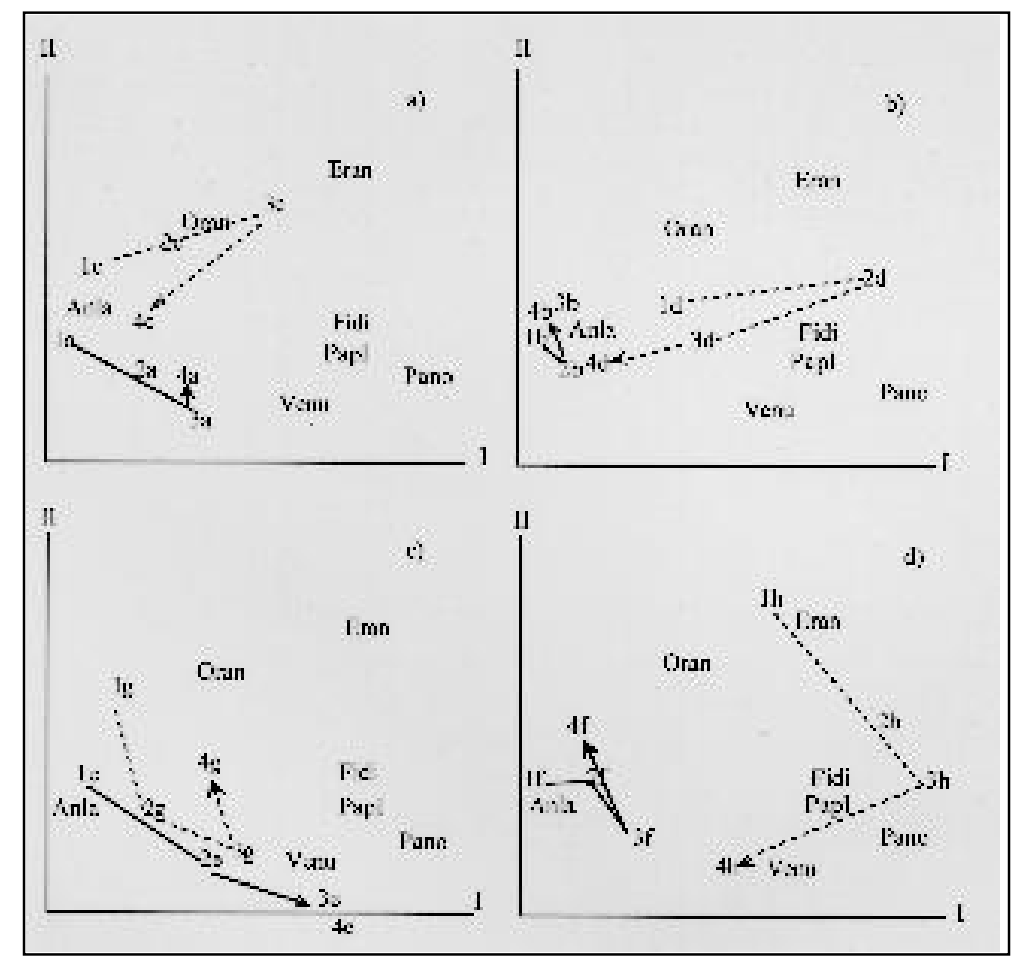

Figura 1 - Trajetórias nos diagramas de ordenação (a até d), em que os números de 1 a 4, representam os inventários de agosto de 1995 a dezembro de 1997 e as letras identificam os tratamentos:

$\mathrm{a}=$ queima e exclusão na encosta; $\mathrm{c}=$ queima e exclusão na baixada; $\mathrm{b}=$ sem queima e exclusão na encosta; $d$ = sem queima e exclusão na baixada; e = queima e pastejo na encosta; $\mathrm{g}=$ queima e pastejo na baixada; $\mathrm{f}=\mathrm{sem}$ queima e pastejo na encosta; $h=$ sem queima e pastejo na baixada. As espécies identificadas segundo as primeiras letras dos epítetos genérico e específico: Andropogon lateralis, Erianthus angustifolius, Fimbrystilis diphylla, Paspalum plicatulum, P. notatum, Orthopapus angustifolius e Vernonia nudiflora, apresentam correlação acima de 0,5 com os eixos de ordenação. O eixo horizontal representa $51,7 \%$ e o vertical $22,2 \%$ da variação total na composição florística. 
apresenta um comportamento semelhante ao do queimado e pastejado (e), ou seja, magnitudes decrescentes de segmentos da trajetória. Ao final da trajetória, pode ter sido alcançado um estado estacionário que reflete a resiliência da vegetação campestre a estes distúrbios, fruto de uma história evolutiva particular. $O$ tratamento pastejado, na encosta (trajetória e), produziu um efeito direcional mais prolongado, que pode ser atribuído apenas ao pastejo ou a uma interação deste com o fogo. Acredita-se que o efeito mais importante é o do pastejo, pois, embora ambos removam área foliar das plantas, e o fogo o faça mais efetivamente, o pastejo continua a reduzir o material verde de lâminas foliares ao longo de toda a estação de crescimento, afetando sua taxa de acumulação (NOY-MEIR, 1995).

A trajetória da parcela queimada e pastejada, na encosta (e), é mais longa em seu primeiro segmento, ou seja, no período de agosto de 95 a janeiro de 96, imediatamente após a aplicação dos tratamentos. Os últimos inventários da área queimada e pastejada praticamente se localizam na mesma posição no plano de ordenação. A redução gradativa na magnitude da alteração da composição entre avaliações sucessivas pode ser atribuída ao fato de a parcela queimada ter sofrido uma intensidade de pastejo bem maior no período de rebrote pósqueimada, quando a proporção de material verde era maior do que no restante do potreiro. Esta capacidade de as áreas recém queimadas representarem um atrativo aos herbívoros também foi observada por WALLACE (1990), KERSTING (1994), DAMÉ et al. (1997) e BRÂNCIO et al. (1997).

Todos os tratamentos excluídos sofreram uma queima acidental em maio de 1997, porém as trajetórias no período dezembro de 1996 a dezembro de 1997 foram curtas, mantendo a composição florística pouco alterada. Este fato reforça a hipótese de resiliência da vegetação a este distúrbio.

Em contrapartida, as parcelas não queimadas na encosta (trajetórias b e f) convergem para um ponto comum ao final do período de avaliação, independente de serem ou não pastejadas. Apresentam uma trajetória quase circular, mantendo-se próximas da composição florística inicial. O pastejo, entretanto, determinou segmentos de trajetória maiores, confirmando a já mencionada importância deste fator de distúrbio.

Ao contrário do observado na encosta, nas trajetórias das parcelas de baixada (Figura 1), destaca- se a convergência das trajetórias $(\mathbf{c}, \mathbf{d}, \mathbf{g}, \mathbf{h})$, tanto nos tratamentos pastejados $(\mathbf{g}, \mathbf{h})$, quanto nos excluídos (c, d), independentemente do fato de terem ou não sido queimados. É possível observar que, apesar da composição inicial bem diversa das parcelas pastejadas, ambas são direcionadas a uma redução da participação de espécies cespitosas como Erianthus angustifolius e Andropogon lateralis e aumento da contribuição de Paspalum notatum e Vernonia nudiflora, espécies rizomatosas. Estas espécies apresentam os pontos de crescimento protegidos ao nível do solo, como rizomas, ou abaixo do solo, como xilopódios.

As parcelas excluídas na baixada (c, d) apresentam trajetórias quase circulares, retornando, em dezembro de 1997, a uma composição próxima da inicial. Com relação à magnitude das trajetórias, pode-se observar que aquelas não queimadas $(\mathbf{d}, \mathbf{h})$, tanto excluídas como pastejadas, tiveram um comprimento maior. Entretanto, na parcela pastejada (h), o afastamento da composição florística inicial é decorrência do pastejo e não do fogo. Esta comparação permite reforçar a hipótese levantada quando da discussão da posição da encosta, de que o pastejo tenha sido o distúrbio com maior capacidade de afetar a dinâmica vegetacional.

Os resultados reforçam a hipótese de resiliência da vegetação campestre ao distúrbio fogo, especialmente no caso das gramíneas, como apontam ANDERSON (1990), SCHÜLE (1990), BOND \& WILGEN (1996). Embora haja referências locais que também possam sustentar esta idéia (EGGERS \& PORTO, 1994; KERSTING, 1994; DAMÉ et al., 1997; PILLAR \& QUADROS, 1997), outros autores indicam a queima como agente modificador da composição florística (FONTANELI \& JACQUES, 1988; DÜRR et al., 1993; COUTINHO, 1994; GARCIA, 1998). Esta diferença de posição pode ser atribuída, ao menos em parte, ao fato de a comparação de áreas queimadas ser feita com outras parcelas que sofreram práticas de melhoramento ou não estarem sob pastejo, nas últimas referências citadas.

Para a avaliação da interação entre os fatores: fogo e pastejo, bem como dos efeitos de cada um deles, foi também realizada uma análise de variância com os dados do último ano.

$\mathrm{O}$ fator pastejo apresentou efeito significativo $(\mathrm{P}=0,077)$ (Tabela 1$)$. Pode-se afirmar que este fator foi determinante da dinâmica da vegetação. Esta observação é compartilhada por COLLINS \& BARBER (1985); BROWN \& STUTH (1993) e TAINTON et al. (1996), entre outros autores. O efeito do fator fogo indicado por uma probabilidade próxima a um nível de significância $(\mathrm{P}=0,094)$ está vinculado às parcelas pastejadas na encosta, que apresentaram trajetórias mais longas 
Tabela 1 - Testes de aleatorização avaliando efeitos de fogo e pastejo e de sua interação sobre a composição de parcelas experimentais de $0,5 \times 3 \mathrm{~m}$, no terceiro ano de avaliação. A análise utilizou distâncias de corda calculadas sobre a biomassa das espécies identificadas.

\begin{tabular}{lcc}
\hline Fontes de variação & Soma de Quadrados & $\begin{array}{c}\text { Probabilidade } \\
\left(\mathrm{SQ}_{\mathrm{entre}}>=\mathrm{SQ}_{\text {dentro }}\right)\end{array}$ \\
\hline Fogo & 0,5512 & 0,0938 \\
Pastejo & 0,9389 & 0,077 \\
Fogo x Pastejo & 0,4034 & 0,2505 \\
\hline
\end{tabular}

(Figura 1, trajetória e). A interação fogo x pastejo não foi significativa $(\mathrm{P}=0,2505)$ sobre a composição da vegetação.

\section{CONCLUSÕES}

Ainda que as hipóteses de resiliência da vegetação campestre ao efeito do fogo e de que o pastejo seja determinante da composição florística possam ser inferidas com base nas avaliações realizadas durante o período experimental, esse não pode ser considerado suficientemente longo de forma a permitir uma maior segurança nas conclusões formuladas. Torna-se necessário utilizar um modelo preditivo que possibilite uma ampliação da escala temporal sobre a qual se realizam as inferências, sugerindo-se o uso de cadeias de Markov.

\section{REFERÊNCIAS BIBLIOGRÁFICAS}

ANDERSON, R.C. The historic role of fire in the North American grassland. In: COLLINS, S.L., WALLACE, L.L. (eds.) Fire in North American tallgrass prairies. Norman : University of Oklahoma, 1990. p.8-18.

BOND, W.J., WILGEN, B.W. van. Fire and plants. London: Chapman and Hall, 1996. 263p. (Population and community biology, 14)

BRÂNCIO, P.A., NASCIMENTO JR., D., REGAZZI, A.J. et al Avaliação de pastagem nativa dos cerrados submetida à queima anual. 1. Composição da dieta de bovinos. Revista Brasileira de Zootecnia,Viçosa, v.26, n.3, p.429-437, 1997.

BROWN, J.R., STUTH, J.W. How herbivory affects grazing tolerant and sensitive grasses in a central Texas grassland: integrating plant response across hierarchical levels. Oikos, Copenhagen, v.67, n.2, p.291-298, 1993.

CASTILHOS, Z.M.S., JACQUES, A.V.A. Caracterização morfológica de espécies nativas e uma cultivada numa pastagem natural submetida a tratamentos de introdução de Trevo vesiculoso cv. Yuchi (Trifolium vesiculosum Savi), ceifa e queima. Porto Alegre : Secretaria da Agr. Est.
Rio Grande do Sul, 1985. v.12, p.115-140. (Anu. Téc. IPZFO).

COLLINS, S.L., BARBER, S.C. Effects of disturbance on diversity in mixed-grass prairie. Vegetatio, The Hague, v.64, p.87-94, 1985.

COUTINHO, L.M. O uso do fogo em pastagens naturais brasileiras. In: PUIGNAU, J.P. Utilizacion y manejo de pastizales. Montevideo, Uruguay : IICA-PROCISUR (Instituto Interamericano de Cooperacion para la Agricultura), 1994. 266p. p.159-168. (DIALOGO XL).

DAMÉ, P.R., QUADROS, F.L.F. de, KERSTING, C.E.B. et al. Efeitos da queima seguida de pastejo ou diferimento sobre a produção, qualidade, cobertura do solo e sistema radicular de uma pastagem natural. Ciência Rural, S. Maria, v.27, n.1, p.133-137, 1997.

DÜRR, J.W., CASTILHOS, Z.M.S., FLORES, A.I.P. et al. Melhoramento da pastagem natural: queima, ceifa, pastejo intenso e adubação como modificadores da composição florística. Revista da Sociedade Brasileira de Zootecnia, Viçosa, v.22, n.2, p.330-340, 1993.

EGGERS, L., PORTO, M.L. Ação do fogo em uma comunidade campestre secundária, analisada em bases fitossociológicas. Porto Alegre : Instituto de Biociências da UFRGS, 1994. 88p. (Boletim do Instituto de Biociências, 53).

FONTANELI, R.S., JACQUES, A.V.A. Melhoramento de pastagem natural: ceifa, queima, diferimento e adubação. Revista da Sociedade Brasileira de Zootecnia, Viçosa, v.17, n.2, p.180-194, 1988.

GARCIA, E.N. Comportamento da vegetação campestre sob diferentes práticas de melhoramento e exclusão. Porto Alegre, 1998. 138p. Dissertação (Mestrado em Zootecnia, Plantas Forrageiras) - Programa de Pós-graduação em Agronomia, Universidade Federal do Rio Grande do Sul, Porto Alegre, 1998.

KERSTING, C.E.B. Avaliação dos efeitos da queima, seguida de pastejo ou diferimento em uma pastagem natural. Santa Maria, RS, 1994. 122p. Dissertação (Mestrado em Zootecnia) - Curso de Pós-graduação em Zootecnia, Universidade Federal de Santa Maria, Santa Maria, 1994.

NABINGER, C. Técnicas de melhoramento de pastagens naturais no Rio Grande do Sul. In: SEMINÁRIO SOBRE PASTAGENS: de que pastagens necessitamos, 1980, Porto Alegre. Anais... Porto Alegre : FARSUL, 1980. p.28-58

NOY-MEIR, I.. Interactive effects of fire and grazing on structure and diversity of Mediterranean grasslands. Journal of Vegetation Science, Uppsala, v.6, p.701-710, 1995.

PILLAR, V.D.P. .Multivariate exploratory analysis and randomization testing with MULTIV. Coenoses, Gorizia, v.12, p.145-148, 1997.

PILLAR, V. De P., ORLÓCI, L. Character-based community analysis: the theory and an application program. The Hague: SPB, 1993. 270p. (Ecological computations series; vol.5). 
PILLAR, V. De P., ORLÓCI, L. On randomization testing in vegetation science: multifactor comparisons of relevé groups. Journal of Vegetation Science, Uppsala, v.7, p.585-592, 1996.

PILLAR, V. De P. , QUADROS, F.L.F. de. Grasslands-forest boundaries in southern Brazil. Coenoses, Gorizia, v.12, n.23, p.119-126, 1997

SCHÜLE, W.. Landscapes and climate in prehistory: interactions of wildlife, man and fire. In: GOLDAMMER, J. G. (ed.) Fire in the Tropical Biota. Berlin: Springer-Verlag, 1990. p.273-318

TAINTON, N.M., MORRIS, C.D., HARDY, M.B. Complexity and stability in grazing systems. In: HODGSON, J., ILLIUS
A.W. (eds.) The ecology and management of grazing systems. Wallingford : CAB International, 1996. p.275-299.

TOTHILL, J.C., HARGREAVES, J.N.G., JONES, R.M. et al. Botanal - A comprehensive sampling and computing procedure for estimating pasture yield and composition. 1 . Field sampling. Tropical Agronomy Technical Memorandum n.7, 24p, 1992.

VALENTINE, J.F. Range development and improvements. Provo : Brigham Young University, 1974. 393p. Cap.6: Range improvement by burning.

WALLACE, L.L. Epilogue: A search for paradigms. In: COLliNS, S.L., WALLACE, L.L. (eds.) Fire in North American tallgrass prairies. Norman : University of Oklahoma, 1990. p.147-151.

Ciência Rural, v. 31, n. 5, 2001. 